\title{
Production of fermented Thai red glutinous rice using an isolated Monascus purpureus NART001 from commercially available Chinese red fermented rice
}

\author{
Preparação de arroz vermelho glutinoso Tailandês fermentado usando Monascus purpureus \\ NART001 isolado do arroz vermelho fermentado Chinês disponivel comercialmente
}

\author{
Ana Silva, João Moreira, Pedro Andrade, Marisa Nicolai, Patrícia Rijo, Nelson Tavares \\ CBIOS - Research Center for Biosciences and Health Technologies, Universidade Lusófona de Humanidade e Tecnologias, \\ Campo Grande 376, 1649-024 Lisboa, PORTUGAL \\ Email: nelson.tavares@ulusofona.pt
}

\begin{abstract}
Red yeast rice is a fermented product used either as food or food supplement to promote blood cir-culation and lower blood cholesterol. Ochratoxin A and citrinin are very important mycotoxins fre-quently found in food products of plant origin. In this work, red yeast rice was prepared from Thai glutinous rice using Monascus purpureus isolated from an available commercial Chinese red yeast rice. A Monascus purpureus NART001 strain was isolated by the surface sterilization method. The strain isolated from the commercially red yeast rice was identified by morphological and taxonomic methods. The ochratoxin A and citrinin content of the fermented red yeast rice previously prepared were evaluated by HPLC-DAD. Moreover, the HPLC analysis included the ochratoxin A and citrinin standards and the red yeast rice samples. The overlay analysis of the mycotoxins chromatograms and UV-VIS spectra indicated that these compounds were not detected in the analyzed extracts under the employed conditions. Further studies with Monascus purpureus NART001 are currently underway.
\end{abstract}

Keywords: Monascus purpureus; Red yeast rice, Ochratoxin A, Citrinin.

\section{Resumo}

A levedura de arroz vermelho é um produto fermentado utilizado como alimento ou suplemento alimentar para promover a circulação do sangue e reduzir o colesterol no sangue. A ocratoxina A e a citrinina são micotoxinas muito importantes frequentemente encontrados em produtos alimentares de origem vegetal. Neste trabalho, a levedura de arroz vermelho foi preparada a partir de arroz glutinoso Tailandês usando o Monascus purpureus isolado do arroz vermelho fermentado Chinês comercialmente disponível. O fungo Monascus purpureus NART001 foi isolado pelo método de esterilização à superfície. O isolado obtido foi identificado por métodos morfológicos e taxonómicos. A ocratoxina A e citrinina contidas no arroz vermelho fermentado, preparado anteriormente, foram avaliados por HPLC-DAD. Além disso, a análise HPLC incluiu os padrões de ocratoxina A e citrinina e as amostras de arroz vermelho fermentado. A análise de sobreposição dos cromatogramas de micotoxinas e espectros UV-VIS permitiram demonstrar que estes compostos não foram detectados nos extractos analisados, sob as condições empregues. Estão sendo realizados novos estudos com Monascus purpureus NART001.

Palavras-Chave: Monascus purpureus, Arroz vermelho fermentado, Ocratoxina A, Citrinina. 


\section{Introduction}

Red yeast rice is a product obtained from rice fermented by a red yeast, Monascus purpureus. This type of rice has been recognized as early as 800 A.D., during the Tang Dynasty in China. (1). It has been used for centuries in Chinese cuisine, as medicine for treating digestive disorder and as medicinal food to promote blood circulation. In some Asian countries, red yeast rice is a dietary staple, and it has a long history of being used as a preservative and natural dye for foods (1). This rice continues to be widely used today in red yeast rice wine production and as a dye and preservative in the food industry $(2,3)$. Glutinous rice is a type of rice that grows and is widely consumed in Southeast and East Asia, especially in Thailand. It has opaque grains, very low amylose content and high amylopectin content, reaching $95 \%$, which contributes to a sticky appearance. The difference in their main composition may affect the content of useful compounds in fermented products and the properties of those products (4). The medicinal properties of red yeast rice have a favorably impact on lipid profiles of hypercholesterolemic patients $(2,3)$. In 2011, a European Food Safety Authority (EFSA) report confirms that monacolin $\mathrm{K}$ present in the red yeast rice contributes to reduction of serum cholesterol levels, especially LDL cholesterol (5). Procedures for preparation of red yeast rice extract were previously described by many authors $(6,7,8,9)$. However, the often co-existing ochratoxin A and citrinin, nephrotoxic and hepatotoxic metabolites that causes the concerns for safety, were frequently found in some food products of animal and plant origin. The mycotoxin citrinin was detected in foodstuffs of vegetable origin (e.g. cereals), food supplements based on rice fermented with red microfungi Monascus purpureus and in foodstuffs of natural origin (10). Ochratoxin A is another toxic and potentially carcinogenic fungal toxin found in a variety of food commodities, including cereals and cereal products. The assessment of citrinin and ochratoxin A in foodstuffs is important because both toxins are considered health risk factors.

The main objective of the present study was the isolation of Monascus purpureus from commercial Chinese red yeast rice and the preparation of glutinous red yeast rice using Thai glutinous rice Oryza sativa L. from the isolated strain. A preliminary investigation was also carried out in order to detect the occurrence of citrinin and ochratoxin A in the prepared red yeast rice from Thai glutinous rice by HPLC.

\section{Introdução}

$\mathrm{O}$ arroz vermelho fermentado é um produto obtido a partir do fungo Monascus purpureus. Este tipo de arroz tem sido utilizado desde 800 D. C., popularizado durante a dinastia Tang, na China. (1). Tem sido largamente usado na cozinha chinesa, também como medicamento para o tratamento de distúrbios digestivos, durante séculos, e como medical food para promover a circulação sanguínea. Em alguns países asiáticos, o arroz vermelho é um alimento básico e tem sido usado ao longo dos tempos como corante e conservante natural para alimentos (1). Hoje em dia, o arroz continua a ser amplamente utilizado na produção de vinho de arroz fermentado e tem tido amplamente uso como corante e conservante na indústria alimentar $(2,3)$. $\mathrm{O}$ arroz glutinoso é outro tipo de arroz que cresce e é abundantemente consumido no Sudeste e Leste da Ásia, especialmente na Tailândia. Este arroz possui grãos opacos, baixo teor de amilose e alto teor de amilopectina, atingindo por vezes $95 \%$, o que contribui para uma aparência gomosa. As variações na composição podem afetar os conteúdos fermentados e consequentemente as propriedades dos referidos produtos (4). As propriedades medicinais do arroz vermelho fermentado têm um impacto favorável a nível do perfil lipídico de pacientes com hipercolesterolemia (2, 3). Em 2011, um relatório da Autoridade Europeia para a Segurança dos Alimentos (EFSA) confirmou que a monacolina $\mathrm{K}$ presente no arroz vermelho fermentado contribui para a redução dos níveis de colesterol sérico, especialmente o colesterol LDL (5). Procedimentos para a preparação de extrato de arroz vermelho fermentado foram previamente descrito por vários autores $(6,7,8,9)$. Contudo, a ocratoxina A e citrinina, micotoxinas nefrotóxicas e hepatotóxicas que co-existem em muitos casos, são frequentemente identificados em alguns produtos alimentares de origem animal e/ou vegetal, fazendo com que as preocupações a nível de segurança aumentem. A micotoxina citrinina foi detectada em alimentos de origem vegetal (por exemplo, cereais), nos géneros alimentícios de origem natural e em suplementos alimentares à base de arroz vermelho fermentados com o microfungo vermelho Monascus purpureus (10). A ocratoxina A é outra toxina fúngica tóxica e potencialmente cancerígena encontrada numa variedade de produtos alimentares, incluindo cereais e produtos cerealíferos. A exposição a citrinina e ochratoxina A é de grande relevância para a avaliação de riscos para a saúde. Deste modo, é necessário analisar a existências destas micotoxinas para evitar os efeitos nocivos resultantes do consumo de alimentos contaminados.

Em conclusão, o principal objetivo do presente estudo é 


\section{Materials and Methods}

Chinese red yeast rice and commercial Thai glutinous rice, Oryza sativa L., were used to obtain the Monascus purpureus strain. Ochratoxin A and citrinin were obtained from Alfa Aesar (Karlsruhe, Germany). All chemicals were of analytical grade.

\section{Isolation of Monascus purpureus}

Surface sterilization method was used to eliminate the contaminants as previously described (6). The rice was first soaked for 1 minute in $95 \%$ ethanol, then washed with a $0.1 \%$ sodium hypochlorite solution for 4 minutes, and finally rinsed with $95 \%$ ethanol for $30 \mathrm{sec}-$ onds. Four washed red rice grains were placed on Rose Bengal agar plates. One week culture was used for the isolation of uncontaminated grains by the cultivation on potato dextrose agar (PDA). The culture was preserved in a refrigerator at $5^{\circ} \mathrm{C}$ as a stock culture.

\section{Identification of Monascus purpureus}

As previously described (11), the NART001 strain, isolated from commercially available red yeast rice, was identified by conventional methods and taxonomic investigations. The strain was inoculated on potato dextrose agar (PDA) and incubated at room temperature to observe morphological characteristics, including colonies, ascospores and conidial stage. Pigment production was also observed. The starch hydrolyzing enzyme activity was studied by cultivation of the strain on medium followed by clear zone detection using iodine solution (6).

\section{Preparation of red yeast rice}

Inoculation and cultivation of isolated Monascus purpureus NART001 were performed using glutinous rice Oryza sativa L. The controlled conditions with appropriate weight, culture age, inoculation volume, temperature, humidity and $\mathrm{pH}$ were adapted from previous studies $(6,7,8,9)$. Samples after 3 -week cultivation were then retained for further use. Glutinous rice grains were immersed in water for 6 hours following by a steaming of 20 minutes. After cooling, $50 \mathrm{~g}$ of steamed rice were placed in a $250 \mathrm{~mL}$ flask and sterilized at $15 \mathrm{psi}$ and $121^{\circ} \mathrm{C}$ for 15 minutes. One week old precultured Monascus purpureus NART001 was used as inoculum. The inoculated rice was incubated at $30^{\circ} \mathrm{C}$ for 3 weeks, after which it was dried in the oven at $65^{\circ} \mathrm{C}$ for 6 hours to obtain the final dried red yeast rice. o isolamento do fungo Monascus purpureus a partir do arroz vermelho fermentado Chinês disponível comercialmente e a preparação de arroz vermelho glutinoso preparado a partir dos grãos de arroz glutinoso Tailandês Oryza sativa L.. Foi igualmente realizado um estudo preliminar cuja finalidade foi detectar por HPLC-DAD a ocorrência das micotoxinas citrinina e ocratoxina $A$ no extracto obtido a partir do arroz vermelho glutinoso fermentado.

\section{Materiais e Métodos}

O arroz vermelho fermentado Chinês comercial e o arroz glutinoso Tailandês Oryza sativa $\boldsymbol{L}$. foram usados para obter o fungo Monascus purpureus. A ocratoxina A e a citrinina foram adquiridas a Alfa Aesar (Karlsruhe, Alemanha). Todos os reagentes usados são de grau analítico.

\section{Isolamento de Monascus purpureus}

O método de esterilização de superfície foi utilizado para eliminar os contaminantes, como anteriormente descrito (6). O isolamento foi efectuado colocando o arroz vermelho Chinês em etanol a $95 \%$ durante $1 \mathrm{mi}-$ nuto e lavando com uma solução de hipoclorito de sódio a $0,1 \%$, durante 4 minutos e, finalmente, lavando com etanol a 95\%, durante 30 segundos. Os grãos de arroz, lavados quatro vezes, foram posteriormente colocados em placas de Agar Rosa de Bengala. Após uma semana a cultura foi utilizada para o isolamento de grãos não contaminados para a cultura do fungo em placas com Agar de Dextrose e Batata (PDA). A cultura foi mantida num frigorífico a $5{ }^{\circ} \mathrm{C}$ como uma cultura stock.

\section{Identificação de Monascus purpureus}

Tal como descrito anteriormente (11), o fungo Monascus purpureus NART001, isolado a partir de levedura de arroz vermelho disponível comercialmente, foi identificado por métodos convencionais e estudos taxonómicos. O fungo foi inoculado em PDA e incubado, à temperatura ambiente, para observar características morfológicas como as colónias, ascosporos e estádio conidial. Também foi observada a produção de pigmento. A actividade da enzima que hidrolisa o amido foi estudada por cultura da estirpe num meio rico em amido, seguida por detecção de zona clara usando solução de iodo (6).

\section{Preparação do Arroz Vermeho Fermentado}

O fungo Monascus purpureus NART001 isolado foi utilizado para a inoculação e cultura do fungo, sendo realizadas usando arroz glutinoso Oryza sativa L. As 
High-performance liquid chromatography analysis (HPLC)

The presence of both mycotoxins was performed by High-Performance Liquid Chromatography (HPLC) analysis, using an Agilent Technologies 1200 Infinity Series LC System equipped with diode array detector (DAD), ChemStationSoftware and a Hypersil ODS column $(250 \mathrm{~mm} \times 4.0 \mathrm{~mm}, 5 \mu \mathrm{m}$ particle size $)$. Samples for analysis ( $1 \mathrm{mg}$ of end-product per $1 \mathrm{~mL}$ of $70 \%$ ethanol) were sonicated for 1 hour and centrifuged for 10 minutes. Extracts were filtered and analyzed by injection of $20 \mu \mathrm{L}$ of each sample and using a gradient composed of acetonitrile (solution A) and trichloroacetic acid (solution $\mathrm{B}$ ) : 0 min, 5\% $\mathrm{A}$ and $95 \% \mathrm{~B} ; 15 \mathrm{~min}$, $75 \% \mathrm{~A}$ and $25 \% \mathrm{~B} ; 20 \mathrm{~min}, 75 \% \mathrm{~A}$ and $25 \% \mathrm{~B}$ and 30 $\mathrm{min}, 75 \% \mathrm{~A}$ and $25 \% \mathrm{~B}$. The flow rate was set at $1 \mathrm{~mL} /$ $\mathrm{min}$. The standards solutions $(0.2 \mathrm{mg} / \mathrm{mL})$ were analysed under the same conditions in methanol, and the detection was carried out between 200 and $600 \mathrm{~nm}$. All analyses were performed in triplicate.

\section{Results}

The main morphological and cultural characteristics of the Monascus purpureus strain NART001 are shown in Figures 1-4. The culture became orange with a white edge and the colonies were volcano shaped, slightly higher in their centers (Figure 1). In PDA medium, the red pigment production could be observed (Figure 2). Starch hydrolysis activity was shown by clear zone detection using iodine solution on a Potato Dextrose agar plate (Figure 3). Various stages of fungal formation could be observed. The new isolated strain formed oval aleuroconidia, globose perithecia, and some of the hyphae were filled up with lipid droplets or pigments (Figure 4).

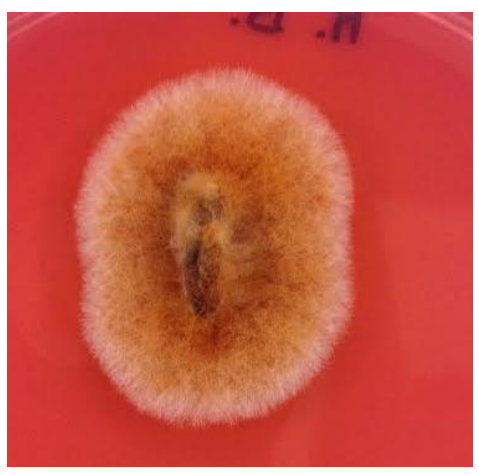

Figure 1/ Figura 1 - Monascus purpureus colony in Rose Bengal Agar plate.

Colónia de Monascus purpureus em placa de Agar Rosa de Bengala. condições como a quantidade, o volume de inoculação, a temperatura, o tempo e $\mathrm{pH}$ foram controladas e adaptadas às condições descritas em estudos anteriores $(6,7$, 8,9 ). As amostras, após três semanas de cultivo, foram armazenadas para posterior utilização. Os grãos de arroz glutinoso foram imersos em água, durante 6 horas, seguido de vaporização durante 20 minutos. Após arrefecimento, $50 \mathrm{~g}$ de arroz vapor foi colocado num frasco de $250 \mathrm{~mL}$ e esterilizado, a 15 psi e $121^{\circ} \mathrm{C}$, durante 15 minutos. Após uma semana o fungo Monascus purpureus NART001 anteriormente isolado foi usado como inóculo. $\mathrm{O}$ arroz inoculado foi incubado a $30^{\circ} \mathrm{C}$, durante 3 semanas. O produto final foi colocado no forno a $65^{\circ} \mathrm{C}$, durante 6 horas, por forma a se obter o arroz vermelho fermentado seco.

\section{Análise por cromatografia liquida de alta performance}

Para a análise de ambas as micotoxinas recorreu-se à cromatografia líquida de alta performance (HPLC). Esta etapa foi realizada num aparelho Agilent Série 1200 Infinito Sistema LC equipado com um detector DAD, controlado com software ChemStation e uma coluna Hypersil ODS (250 mm de diâmetro x 4,0 mm, de $5 \mu \mathrm{m}$ ). As amostras para análise (1 $\mathrm{mg}$ do produto final por $1 \mathrm{~mL}$ de etanol a 70\%) foram colocadas no ultra-sons durante 1 hora e centrifugadas durante 10 minutos. Os extractos foram analisados através da injecção de $20 \mu \mathrm{L}$ de cada amostra e utilizando um gradiente composto de Solução A (acetonitrilo) e a solução B (ácido tricloroacético): 0 min., $5 \%$ de A e $95 \% \mathrm{~B} ; 15 \mathrm{~min} ., 75 \% \mathrm{~A}$ e $25 \% \mathrm{~B} ; 20 \mathrm{~min} ., 75 \%$ A e $25 \%$ B e $30 \mathrm{~min} ., 75 \%$ A e $25 \%$ de B. O caudal foi de $1 \mathrm{~mL} / \mathrm{min}$. Os padrões foram corridos sob as mesmas condições, em metanol, e a detecção foi realizada entre 200 e $600 \mathrm{~nm}$ com um detector DAD. Todas as análises foram realizadas em triplicado.

\section{Resultados}

As principais características morfológicas e culturais são mostradas nas Figuras de 1-4. Colónias da estirpe NART001 de Monascus purpureus adquiriram coloração laranja com bordas brancas com uma elevação central e aspeto em forma de lava (Figura 1). Na placa de Agar de Dextrose e Batata pode ser observada a produção de pigmento vermelho (Figura 2). A atividade de hidrólise do amido mostra-se por detecção da zona clara usando a solução de iodo (Figura 3). Várias fases de formação de fungos pode ser observado (Figura 4). A nova estirpe isolada apresenta aleurconídio oval, peripécia globosa e algumas hifas são preenchidas com gotículas lipídicas ou pigmentos (Figura 4).

A análise de HPLC-DAD foi feita por comparação dos tempos de retenção dos picos dos padrões e amostras e 


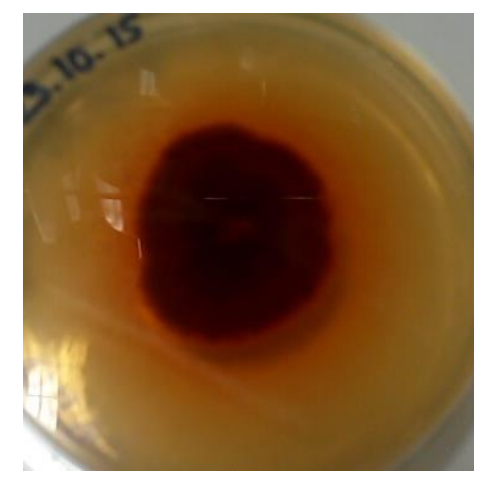

Figure 2/ Figura 2 - Red halo around Monascus purpureus colony in Potato Dextrose Agar plate. Halo vermelho envolvendo a colónia de Monascus purpureus em placa de Agar de Dextrose e Batata.

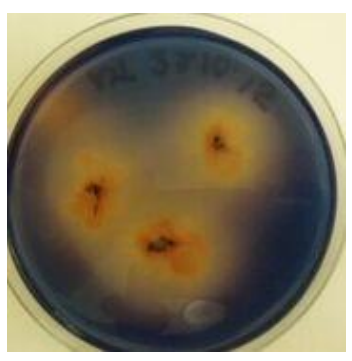

Figure 3/ Figura 3 - Starch hydrolysis activities of Monascus purpureus strain NART001 after two weeks in Potato Dextrose Agar plate.

Atividade hidrolítica de Monascus purpureus NART001 após duas semanas em placas de Agar de Dextrose e Batata.
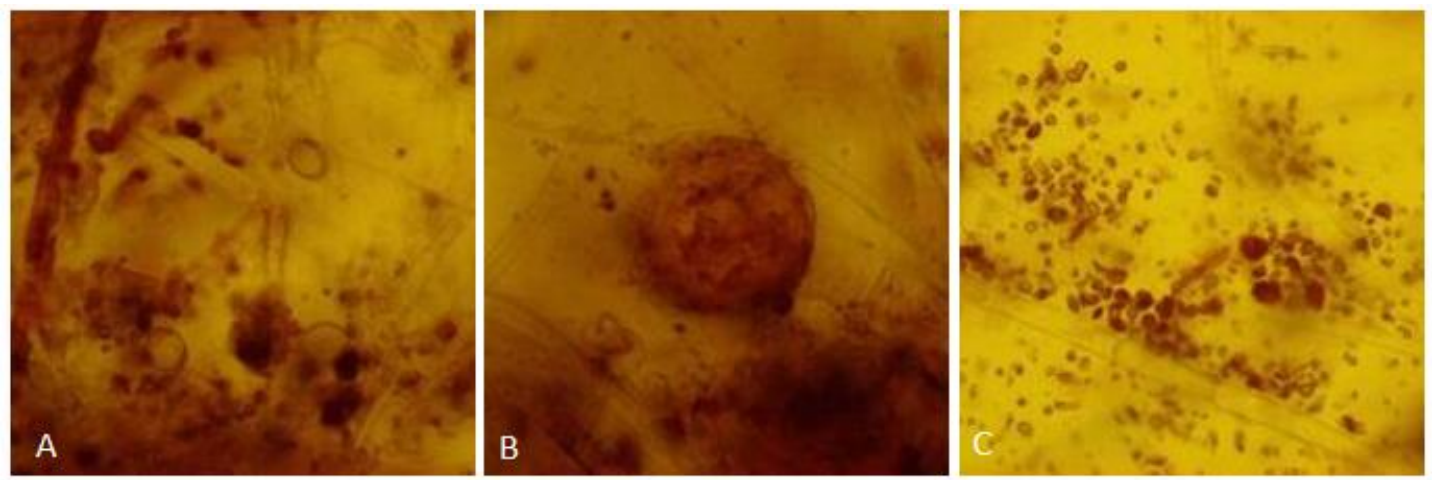

Figure 4/ Figura 4 - Formation of aleucoronidia (A) perithecia (B) and pigment and lipid drops (C) in mycelium of Monascus purpureus strain NART001 (fotografias de microscopia 600x)

Formação de Aleucoronídia (A) peritecia (B) pigmentos e gotículas de gordura (C) no micélio da estirpe NART001 de Monascus purpureus (600x microscope photography) 


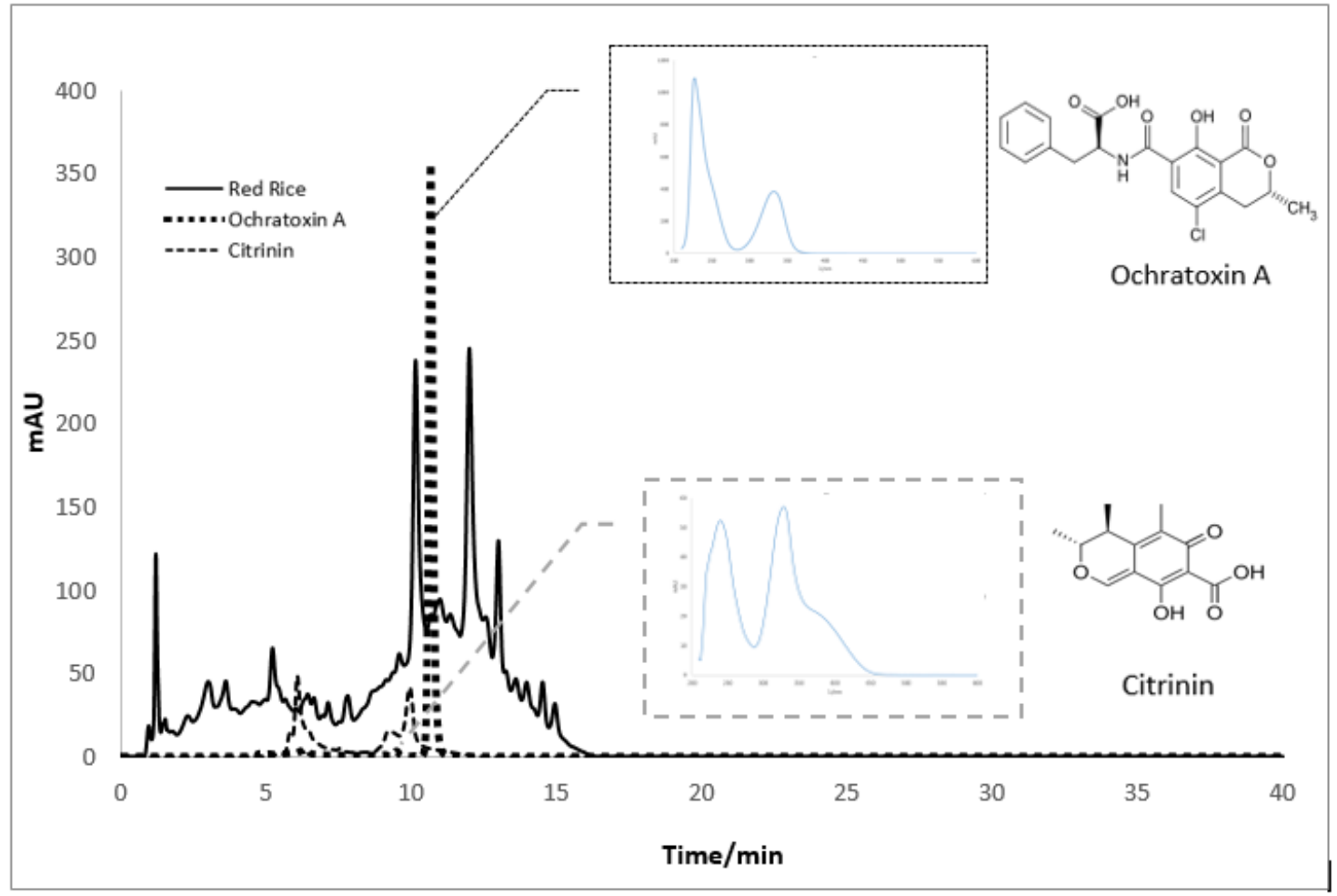

Figure 5/ Figura 5 - HPLC chromatogram (254 nm) and UV-VIS spectra (insert) of ochratoxin A, citrinin and red fermented rice.

Cromatograma obtido por HPLC (254 nm) e espectros UV-VIS de ocratoxina A, citrina e arroz vermelho fermentado.

HPLC-DAD analysis was made by comparing retention times of standards and samples peaks and by overlapping the UV-VIS spectra of pure compounds with UVVIS spectra of samples (Figure 5).

\section{Discussion}

A Monascus purpureus NART001 strain was successfully isolated by surface sterile technique, as observed in Figure 1, 2 and 3. The macroscopic characteristics, such as color and colony shape, and microscopic features, including the presence of aleucoronidia, perithecia, pigment and lipid drops in the mycelium of the Monascus purpureus NART001 strain colonies show similarity to Monascus purpureus, as expected (11). Under the conditions used, it was possible to prepare red yeast rice using Thai glutinous rice from the isolated strain Monascus purpureus NART001 previously obtained from a commercial Chinese red yeast rice sample. sobrepondo os espectros UV-VIS dos compostos puros com espectros UV-VIS de amostras (Figura 5).

\section{Discussão}

A estirpe de Monascus purpureus NART001 foi isolada por técnica estéril superfície, como se pode observar nas Figuras 1-3. As características macroscópicas como a cor e formas das colónias e as microscópicas, incluindo a presença de aleucoronidia, perithecia, pigmento e gotículas lipídicas no micélio da estirpe Monascus purpureus NART001 apresentam similaridade com Monascus purpureus, como era expectável (11). Foi possível obter, nas condições utilizadas, levedura de arroz vermelho preparada a partir de arroz glutinoso Tailandês usando o Monascus purpureus isolado do arroz vermelho fermentado Chinês comercialmente disponível. Pelo método usado, os extractos de etanol de todas as amostras após análise HPLC sugerem que a ocratoxina A e a citrinina estão em quantidade não detectável. Es- 
The ethanol extracts of all samples after HPLC analysis suggest that the levels of ochratoxin A and citrinin are undetectable by the methods used in this study.

Further studies with Monascus purpureus NART001 are underway and with the intention to perform a different fungal culture media in order to optimize the growth. The DNA identification of the strain will be also performed, using a DNA sequencing analysis for molecular species identification. Red pigment content of the red yeast rice extract should be measured by determination of absorbance at $500 \mathrm{~nm}$, which is the maximum wavelength for red color. Determination of monacolin $\mathrm{K}$ content should be performed, considering that this compound is present in red yeast rice. Monacolin $\mathrm{K}$ has medicinal properties that favorably impact lipid profiles of hypercholesterolemia (5). The detection and quantification of the mycotoxins ochratoxin A and citrinin should be carried out after purification processes of the samples.

\section{Conflict of Interest}

The authors declare that there is no financial or personal relationship that can be understood as representing a potential conflict of interest. tudos adicionais com Monascus purpureus NART001 estão a ser realizados e que está previsto utilizar meios de cultura diferentes, a fim de optimizar o crescimento. A identificação de ADN da estirpe deve também ser realizada, usando uma análise de sequenciamento de DNA que atualmente é o método mais utilizado, para a identificação da espécie a nível molecular. O teor de pigmento vermelho do extracto de levedura de arroz vermelho deve ser medido por determinação da absorvância a 500 nm, que é o comprimento de onda máximo para a cor vermelha. Deve ser realizada a determinação do teor de monacolina $\mathrm{K}$, considerando-se que este composto está presente nalevedura de arroz vermelho. A monacolina $\mathrm{K}$ tem propriedades medicinais que promovem um impacto favorável no perfil lipídico da hipercolesterolemia (5). A detecção e quantificação de micotoxinas ocratoxina A e citrinina, deve ser levada a cabo depois dos processos de purificação das amostras.

\section{Conflito de interesses}

Os autores declaram que não existem quaisquer relações pessoais ou financeiras que possam ser consideradas como um potencial conflito de interesses. 


\section{References/Referências}

[1] Marley E., Brown P., Leeman D. and Donnelly C., Analysis of citrinin in cereals, red yeast rice dietary supplement, and animal feed by immunoaffinity column cleanup and LC with fluorescence detection, Journal of AOAC Int., 2016, 99(4):1025-31.

[2] Li Y.G., Liu H., and Wang Z.T., A validated stability-indicating HPLC with photodiode array detector (PDA) method for the stress tests of Monascus purpureus fermented rice, red yeast rice, J. Pharm Biomed Anal., 2005; 39: 82-90.

[3] Hong M, Seram P, Zang Y, Heber D. Anticancer Effects of Chinese Red Yeast Rice Versus Monacolin K Alone on Colon Cancer Cells Journal of Nutritional Biochemistry 2008; 19:448-458.

[4] Insomphun S., Rice (Oryza sativa L.), Faculty of Agriculture, Chiang Mai University, Thailand, 2003, (http// agronomy.agri.cmu.ac.th/ elearning/ agron313/rice. doc, Accessed 17 April 2016)

[5] EFSA Panel on Dietetic Products, Nutrition and Allergies. "Scientific Opinion on the substantation of health claims related to monacolin $\mathrm{K}$ from red yeast rice and maintenance of normal blood LDL-cholesterol concentrations”. EFSA Journal 2011, 9(7): 2304
[6] Chairote E, Chairote G, Wongpornchai S, Lumyong S. Preparation of red yeast rice using various Thai glutinous rice and Monascus purpureus CMU001 isolated from commercial Chinese red yeast rice sample. KMITL Sci. Tech. J., 2007; 7: 28-37.

[7] Yeap S, Beh B, Kong J, et al., In vivo Hypercholesterolemic of MARDI Fermented Yeast Rice Water Extract in High Cholesterol Diet Fed Mice. Evidence-Based Complementary and Alternative Medicine 2014; http://dx.doi. org/10.1155/2014/707829, Acessed 30 April 2016.

[8] Bunnoy A, Saenphet K, Lumyong S, et al., Monascus purpureus-fermented Thai glutinous rice reduces blood and hepatic cholesterol and hepatic steatosis concentrations in diet-induced hypercholesterolemic rats. BMC Complement Altern Med 2015; 15: 88. doi:10.1186/s12906-015-0624-5.
[9] Chairote E, Jannoey P, Chairote G. Improvement of Monacolin K and Minimizing of Citrinin Content in Korkor 6 (RD6) Red Yest Rice.World Academy of Science, Engineering and Technology, International Science Index 97, International Journal of Biological, Biomolecular, Agricultural, Food and Biotechnological Engineering, 2015; 9: 43-46.

[10] Ostry V, Malir F, Ruprich J. Producers and Important Sources of Ochratoxin A and Citrinin. Toxins 2013; 5: 1574-1586; doi: 10.3390/toxins5091574

[11] Rasheva T, Hallet JN, Kujumdzieva A. Taxonomic investigation of Monascus purpureus 94-25 strain. Journal of Culture collections, 1998; 2: 51-59. 